\title{
In Situ Generation of Ru-Based Metathesis Catalyst. A Systematic Study
}

\author{
Daniel S. Müller, ${ }^{\mathrm{a}}$ Yann Raoul, ${ }^{\mathrm{b}}$ Jérôme Le Nôtre, ${ }^{\mathrm{c}}$ Olivier Basléa, ${ }^{\mathrm{a}}$ and Marc Mauduit ${ }^{\mathrm{a} *}$ \\ ${ }^{a}$ Univ Rennes, Ecole Nationale Supérieure de Chimie de Rennes, CNRS, ISCR UMR 6226, F-35000 Rennes, France \\ b OLEON SAS, Venette BP 20609, 60206 Compiègne Cedex, France \\ ${ }^{\text {c }}$ PIVERT SAS, Rue les Rives de l’Oise CS50149, 60201 Compiègne Cedex, France
}

KEYWORDS: Olefin metathesis, Ruthenium, Arene complexes, in situ, NHC ligand

\begin{abstract}
A practical and cost-effective protocol for the in situ generation of Ru-based metathesis catalysts was developed. Assembly of commercially available and inexpensive reagents $\left[\mathrm{Ru}(p \text {-cymene }) \mathrm{Cl}_{2}\right]_{2}, \mathrm{SIPr}$. $\mathrm{HCl}$ and $n$-BuLi led to the formation of 18 electron arene-ruthenium complexes that, in the presence of additives such as alkynes, cyclopropenes and diazoesters, generated highly selective and efficient catalytic systems applicable to a variety of olefin metathesis transformations. Notably, we were able to achieve a productive TON of 4500 for the self-metathesis of methyl oleate, a reaction which could be easily upscaled to $2 \mathrm{~kg}$.
\end{abstract}

Since its discovery in the mid 1950's, the metathesis reaction gained significant importance for organic syntheses and polymer chemistry. ${ }^{1}$ In its early days, the metathesis reaction relied on ill-defined in situ generated catalysts. Only upon the emergence of well-defined air stable Ru-complexes, such as the Grubbs $1^{\text {st }}$ generation Ru-1a, and the more performant Grubbs $2^{\text {nd }}$ generation Ru-1b containing an $N$-heterocyclic carbene (NHC) ligand, olefin metathesis started to significantly impact organic synthesis. ${ }^{2}$ Since then the field has grown steadily, resulting in numerous applications for both academia and industry. ${ }^{3}$ An early mechanistic study from

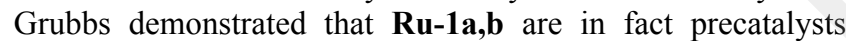
which, only after dissociation of the $\mathrm{PCy}_{3}$ ligand, provide the corresponding catalytically active 14-electron benzylideneruthenium species (Figure 1-A). ${ }^{4}$ Obviously the dissociated ligand (e.g. $\mathrm{PCy}_{3}$ ) is no longer needed in the catalytic cycle and has been shown to even be involved in catalyst decomposition. ${ }^{5}$ In the light of this, several research groups were interested to develop the in situ formation of metathesis active species from alkylidene-free Ru-precatalysts. In that respect, the 18-electron phosphine-based $p$-cymene-ruthenium complex Ru-2, ${ }^{6}$ and its NHC ligated congeners $\mathbf{R u}-\mathbf{3},{ }^{7} \mathbf{R u}-\mathbf{4}^{8}$ and $\mathbf{R u}-\mathbf{5}^{9,10}$ showed metathesis activity in the presence of carbene activator (Figure 1-B). ${ }^{11}$ This in situ protocol was successfully illustrated in ring-opening metathesis polymerization (ROMP), ${ }^{6 a, 12}$ self-metathesis ${ }^{13,14 \mathrm{~d}}$ and ringclosing metathesis (RCM) reactions..$^{14}$ Of note, Thieuleux and Basset demonstrated by ${ }^{1} \mathrm{H}-\mathrm{NMR}$ studies that the selfmetathesis of ethyl oleate with $\mathrm{PCy}_{3}$-based complex Ru-2 and (trimethylsilyl) diazomethane (TMSD) as carbene activator generated the same observable resting states as the Grubbs-1 catalyst (Ru-1). ${ }^{13}$ Moreover, independent studies by Fürstner ${ }^{15}$ and Noels ${ }^{12 d, 16}$ showed that light could also initiate metathesis reactions with 18-electron arene-ruthenium complexes (e.g. Ru-2). More recently, Lewis acid assisted activation of 18electron arene-ruthenium complexes containing various unsaturated NHCs was reported by Beller and coworkers as well as the influence of trace impurities upon metathesis activity. ${ }^{17}$ Despite all these cost-effective methodologies to furnish active metathesis species from simple 18-electron Rucomplexes, few drawbacks merit to be solved to render this protocol more attractive. For instance, the most efficient Ru-5 containing the saturated NHC ancillary ligand (SIMes) is quite unstable and remains difficult to isolate. ${ }^{10,18}$ Inspired by the pioneering work of Grubbs, ${ }^{14 a}$ Noels $^{14 c, 16}$ and Dixneuf ${ }^{14 b}$ we opted for a systematic study to generate 14 electron active species by simply mixing a Ru(II) salt, an ancillary NHC precursors and a carbene activator, thus avoiding any isolation steps of unstable complexes (Figure 1).

\section{- Previous works:}

(A) Formation of 14-electron Ru-active species from well-defined Ru-benzylidene precatalysts by dissociation of $\mathrm{PCy}_{3}$

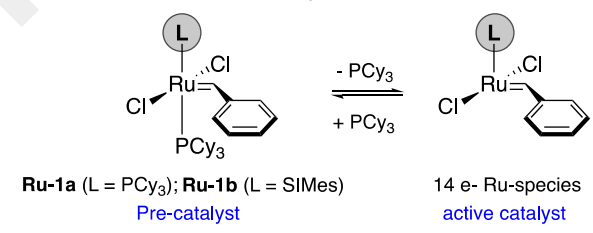

(B) Formation of 14-electron Ru-active species from 18-electron Ru-complexes by dissociation of $p$-cymene in presence of carben source

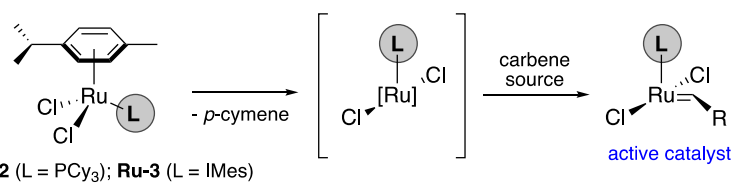

Ru-2 ( $\left.\mathrm{L}=\mathrm{PCy}_{3}\right)$; Ru-3 ( $\left.\mathrm{L}=\mathrm{IMes}\right)$

Ru-4 (L = IPr); Ru-5 ( $\mathrm{L}=$ SIMes)

- This work: In-situ formation of 14-electron Ru-active species from commercially available $\left[(p \text {-cymene }) \mathrm{RuCl}_{2}\right]_{2}$ and azolium $\mathrm{NHC}$ precursors

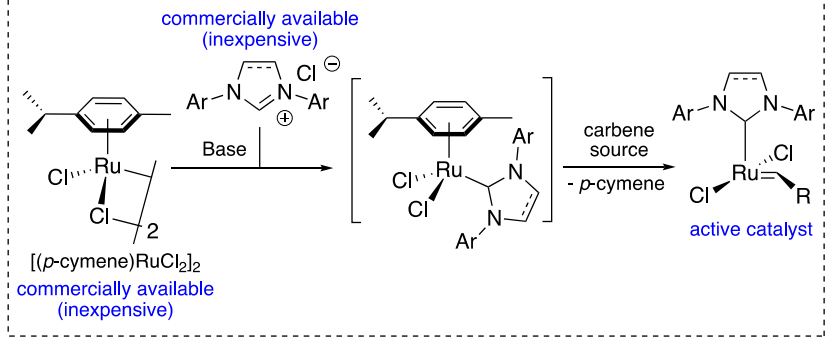

Figure 1. Previous strategies used for the formation of 14-electron Ru-active species toward olefin metathesis (A and B). The proposed synthetic route involving inexpensive commercially available reagents (this work). SIMes $=N, N^{\prime}$-bis-mesityl-imidazolin-2-ylidene 
IMes $=N, N^{\prime}$-bis-mesityl-imidazol-2-ylidene, $\mathrm{IPr}=N, N^{\prime}$-bis-2,6-iPr$\mathrm{C}_{6} \mathrm{H}_{3}$-imidazol-2-ylidene.

We report herein that transient alkylidene $\mathrm{Ru}$-species may be readily formed by mixing inexpensive commercially available $\left[(p \text {-cymene }) \mathrm{RuCl}_{2}\right]_{2},{ }^{19}$ an azolium salt, a base and a carbene activator (Figure 1-this work). The choice of the base and the activator appeared to be pivotal factors in the quest for highly active catalytic species towards the targeted metathesis transformation. Furthermore, the results of this systematic approach led to the development of in situ made rutheniumcatalysts exhibiting catalytic performances that can compete with some well-defined $1^{\text {st }}$ generation catalysts (e.g. Ru-1a).

We began our study by in situ generation of ruthenium arene complex Ru-NHC from four commercially available azolium salts which were deprotonated with NaHMDS and then exposed to $\left[\left(p\right.\right.$-cymene) $\left.\mathrm{RuCl}_{2}\right]$ (Figure 2$) .{ }^{20}$ There is literature precedent on the exchange of $p$-cymene to deuterated benzene and based on this observation, ${ }^{9}$ it is reasonable to assume that $p$-cymene is rapidly replaced by toluene at $80{ }^{\circ} \mathrm{C} .{ }^{21}$ The solution of the in situ generated 18-electron complex Ru-NHC was then immediately tested for the ring-closing metathesis (RCM) of diethyl diallylmalonate $\mathbf{1}$.
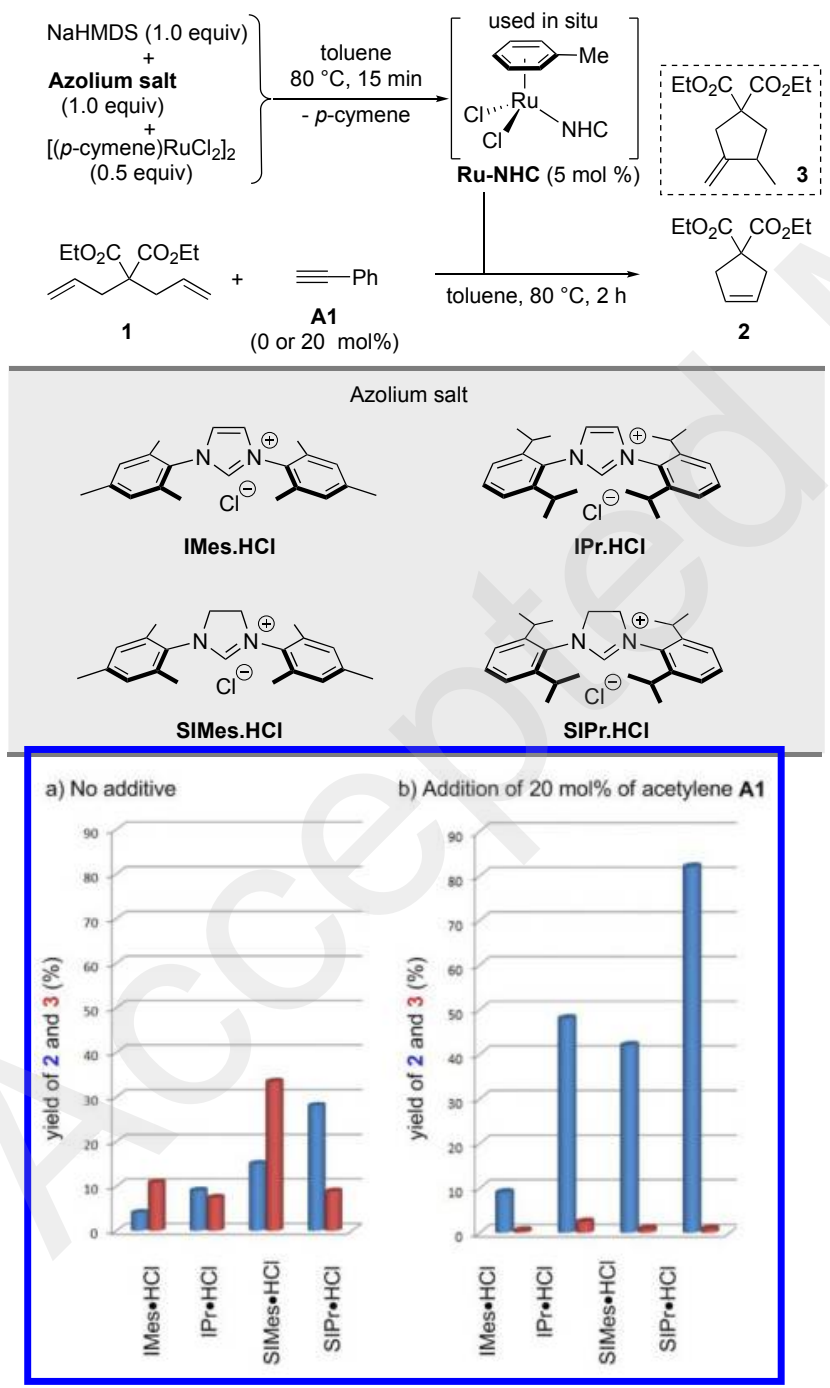

Figure 2. Ratio of RCM-product 2 (blue) and cycloisomerization product 3 (red) in the absence (a) and in the presence (b) of phenyl acetylene A1. $\mathrm{SIPr}=N, N^{\prime}$-bis-2,6- $i \mathrm{Pr}-\mathrm{C}_{6} \mathrm{H}_{3}$-imidazolin-2-ylidene.

This reaction was carried out in the presence (Figure 2-a) and absence (Figure 2-b) of phenyl acetylene $\mathbf{A 1},{ }^{20}$ which has been previously described as a convenient and efficient activator. ${ }^{14 \mathrm{c}, \mathrm{d}}$ In the absence of phenylacetylene, the use of SIPr.HCl salt as NHC precursor afforded the highest selectivity towards the metathesis product 2 (Figure 2-a). In fact, the noticeable activity observed using SIMes ligand precursor led mostly to the cycloisomerization product 3 . Interestingly, in all examined cases a strong enhancement of the metathesis reactivity and selectivity could be observed upon the addition of $20 \mathrm{~mol} \%$ of phenyl acetylene, ${ }^{14 c, d}$ with the SIPr-based complex demonstrating the highest catalytic activity (Figure 2-b). It should be noted that a reduction of the $4: 1$ phenyl acetylene:ruthenium ratio significantly lowered yields and selectivities towards the metathesis product 2 (see Supporting Information; SI). Encouraged by these initial results, we decided to further investigate the influence of the base on the outcome of the metathesis reaction. Therefore, the catalytic activity obtained with $3 \mathrm{~mol} \%$ of SIPr-based complex generated using NaHMDS was compared in terms of efficiency to catalytic systems generated from the use of other bases such as $\mathrm{ZnEt}_{2}$, KHMDS, $n$-BuLi and KOtBu (Figure 3). The catalytic systems generated from the various bases evidenced tremendous metathesis activity differences after 15 min of reaction. Whereas $\mathrm{Et}_{2} \mathrm{Zn}$ gave a very modest $7 \%$ yield of the desired RCM-product 2, NaHMDS and $\mathrm{KO} t \mathrm{Bu}$ gave significantly improved results (37\% and 63\% yield). The best performances were obtained with KHMDS and $n$-BuLi $(\sim 90$ $\%$ GC-yield), which compare favorably to the previous procedure described by Grubbs requiring prolonged reaction times and higher catalyst loading for similar results $(5 \mathrm{~mol} \%$, $10 \mathrm{~h}) .{ }^{14 a}$ With the objective to provide catalytic systems of broad application, we therefore decided to continue our study with $n$-BuLi, which has the advantage of only producing butane and $\mathrm{LiCl}$ side products with low potential for interfering with the metathesis catalytic cycle.

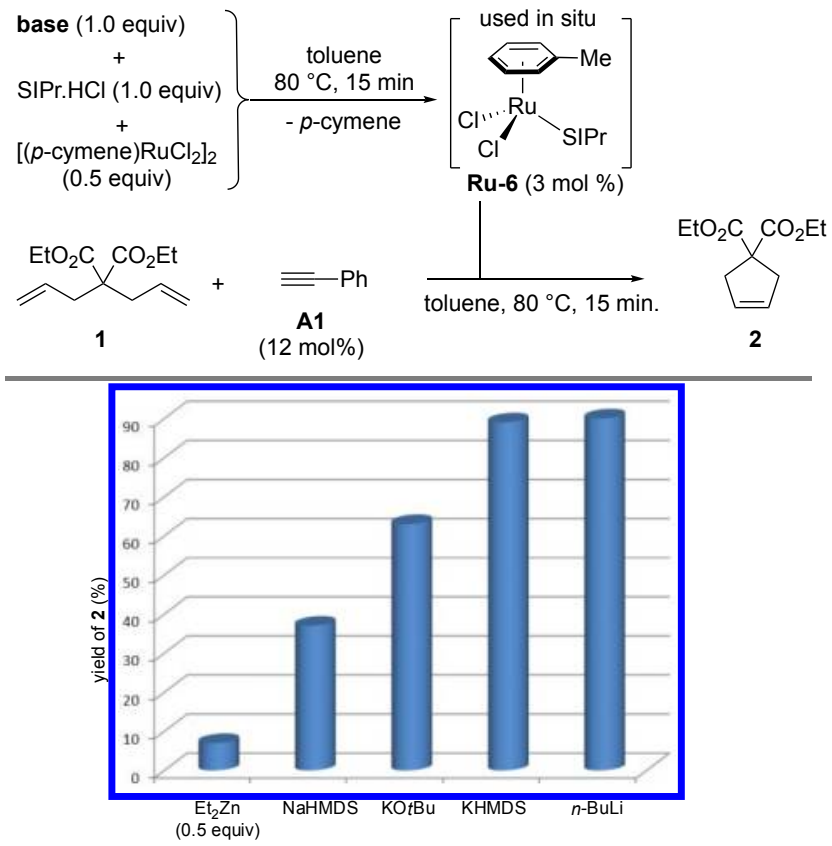


Figure 3. Influence of the base on the catalytic efficiency after 15 $\mathrm{min}$ in the RCM of diethyl diallylmalonate 1 using $3 \mathrm{~mol} \%$ of in situ generated Ru-6. In all cases, the 2:3 ratio was >98:2.

Next, we evaluated and compared the catalytic activity resulting from the use of nine different activators; 3 representatives of each activator type shown in Figure 4. First, the electronic properties of alkyne activators demonstrated an influence on the catalyst activity. While electron neutral or rich alkynes (A1 and $\mathbf{A 2}$ ) afforded the desired RCM product $\mathbf{2}$ in good 87 and $79 \%$ yield, modest $42 \%$ yield was obtained with the electron poor alkyne A3. Then, cyclopropenes additives, which have never been previously considered as activator for 18 electron arene-ruthenium complexes, proved beneficial to the performance of the metathesis reaction. Again, the electronic property of the carbene source is reflected in the catalytic activity: electron rich cyclopropene A4 $^{22}$ generated a metathesis catalytic system, affording product 2 in high $96 \%$ yield, whereas the more electron deficient cyclopropenes $\mathbf{A 5}$ and $\mathbf{A 6}$ gave inferior results. Finally, the addition of diazocompounds such as $\mathbf{A 7}$ or $\mathbf{A 8}$ showed similar activity to produce the RCM product in $70 \%$ yield, whereas TMSD (A9) gave only sluggish reactivity.
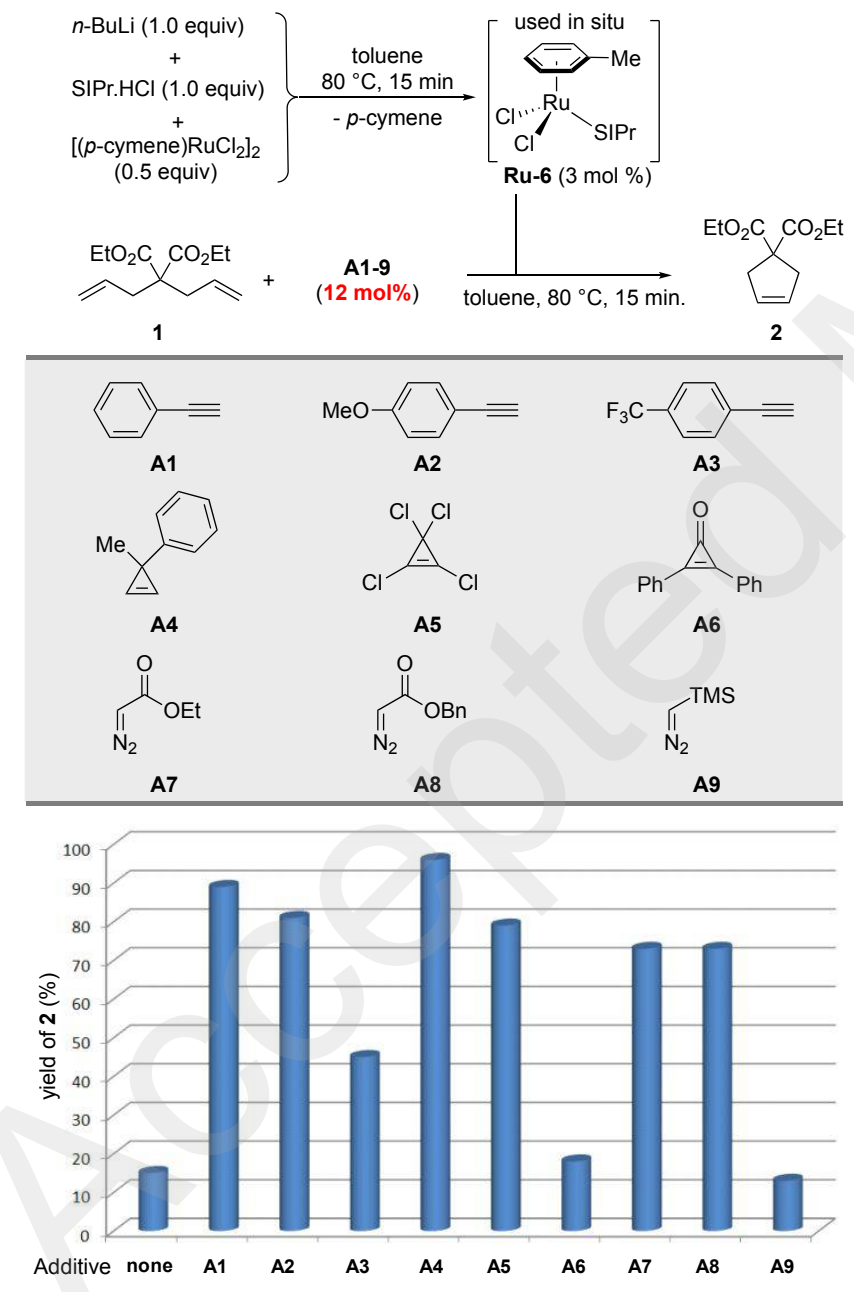

Figure 4. Screening of activators for the RCM of diethyl diallylmalonate 1 .
Concerning the activation of 18 electron arene-rutheniumcomplex Ru-6 with alkyne, cyclopropene and diazo activators we propose the mechanistic scenarios described in Scheme 1. Based on the work of Werner ${ }^{23}$ and $\mathrm{Ozawa}^{24}$ on vinylideneruthenium complexes it was speculated in the past that the addition of alkynes to 18 electron Ru-complexes would lead to vinylidene species as the active catalyst. ${ }^{14}$ However, recent reports by Delaude on homobimetallic ruthenium complexes suggest that the initially generated vinylidene species $\mathbf{C}$ undergoes ene-yne metathesis with excess alkyne to produce after cycloaddition/cycloreversion benyzlidene complex $\mathbf{E}$ which is very likely responsible for the catalytic activity (Scheme 1-a). ${ }^{25}$ This hypothesis is further supported by our experimental findings that an alkyne:Ru ratio inferior to 4:1 resulted in a strong drop of activity and selectivity. ${ }^{20}$ For the reaction with cyclopropenes, we propose the following mechanism (Scheme 1-b). First, thermal decoordination $^{6 \mathrm{~b}}$ of toluene gives the coordinately unsaturated, highly reactive 12 electron ruthenium complex $\mathbf{B}$ which rapidly reacts with cyclopropene $\mathbf{A} 4$ to give the more stable $\pi$-complex $\mathbf{F}$. In fact a recent report by Occhipinti and Jensen described species $\mathbf{B}$ to be responsible for common catalyst degradation by intramolecular $\mathrm{C}-\mathrm{H}$ activation and hydridemechanism isomerization. ${ }^{9}$ Hydride ruthenium species were evoked by Beller as the responsible species for producing cycloisomerization products (e.g. 3; Figure 2). ${ }^{17 \mathrm{a}}$ An alternative isomerization pathway was proposed by Dixneuf who reported that $\mathbf{B}$ can promote cycloisomerization of dienes via formation of a bis- $\pi$-complex followed by oxidative addition, $\beta$-elimination and reductive elimination. ${ }^{14 \mathrm{~b}}$ This is very likely the reason why in the absence of activator the cycloisomerized product $\mathbf{3}$ was formed as the major species (Figure 2). In analogy to the corresponding tungsten complexes we then propose the subsequent formation of metallacyclopropane $\mathbf{G}$ which then rearranges to give the active vinyl alkylidene complex $\mathbf{H}{ }^{26}$ 


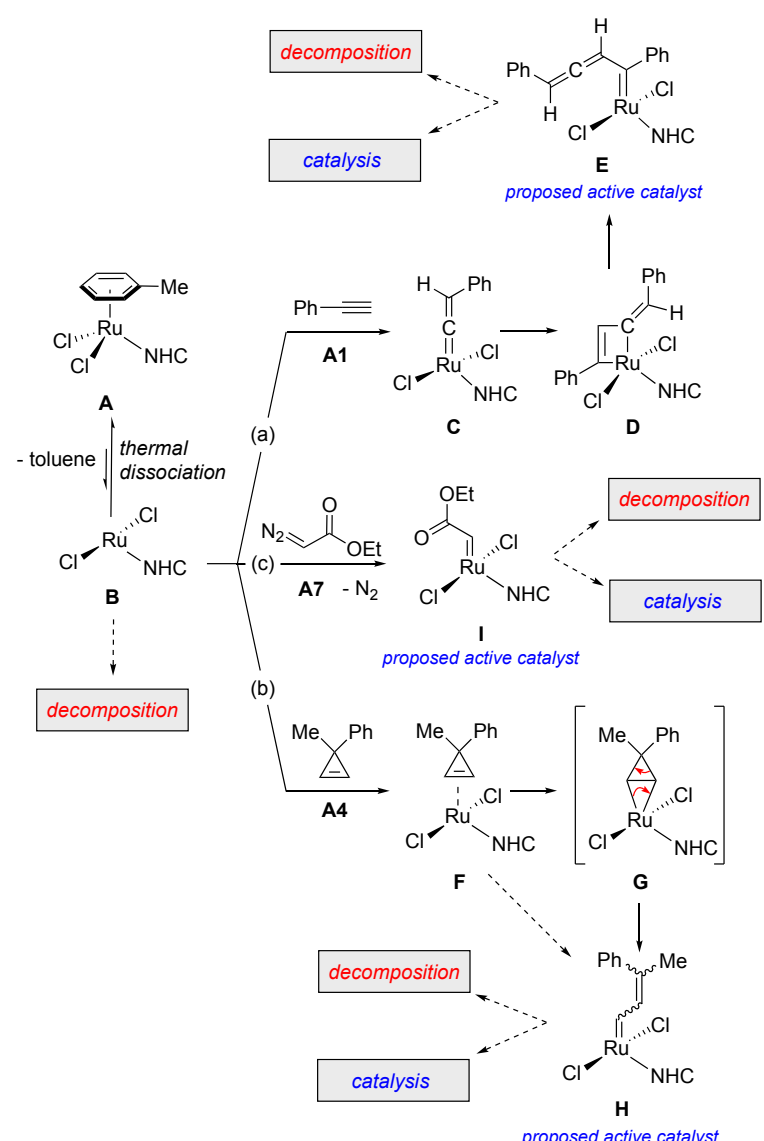

Scheme 1. Postulated mechanisms for the activation with alkynes, cyclopropenes and diazocompounds

Alternatively, the $\pi$-complex $\mathbf{F}$ could also react to $\mathbf{H}$ without formation of the metallacyclopropane intermediate. ${ }^{27}$ Due to the known lack of stability of complex $\mathbf{H}$ we can expect that there will be a partial loss of material due to decomposition. ${ }^{4 \mathrm{~d}}$ Therefore the in situ generated 18-electron complex A needs to be added to the activator and the substrate at the same time. In this case the transiently generated active species $\mathbf{H}$ will immediately enter the catalytic cycle thus diminishing inadvertent decomposition. ${ }^{28}$ According to reactions between $\mathrm{Ru}$-complexes and diazocompounds described in the literature, ${ }^{10,29}$ we propose the formation of $\mathbf{I}$ from $\mathbf{B}$ and $\mathbf{A} 7$ under concomitant nitrogen evolution (Scheme 1-c). Indeed, gas evolution is observed even at room temperature upon addition of A7 to Ru-6.

With the optimized conditions in hand for the in situ generation of the complex Ru-6 that use $n$-BuLi as the base to deprotonate the SIPr ligand precursor, we investigated the substrate scope (Table 1). In order to minimize the risk of potential mismatch between substrates and activators, we decided to evaluate several activators (or no activator) for each of the metathesis reactions. RCM reactions with diallyl- $N$ tosylamine 4 was carried out at $80{ }^{\circ} \mathrm{C}$ and $22{ }^{\circ} \mathrm{C}$ (entries $1-8$ ). At $80{ }^{\circ} \mathrm{C}$ complete conversion was observed with only $2 \mathrm{~mol}$ $\%$ of Ru-6 in combination with activator A1 (entry 2). In contrast, carrying out the same reactions at $22^{\circ} \mathrm{C}$ (entries 5-8) gave similar results only with cyclopropene A4 (entry 7). This indicates that the rate determining step in the in situ formation of active 14-electron ruthenium complexes is very likely not the thermal dissociation of the toluene substituent $(\mathbf{A} \rightarrow \mathbf{B}$; Scheme 1) but rather the formation of $\mathbf{E}$ (Scheme 1-a) in the case of alkynes. Another explanation for the sluggish reactivity with $\mathbf{A 1}$ and $\mathbf{A} 7$ at $22{ }^{\circ} \mathrm{C}$ might be that these catalytic systems require thermal activation. ${ }^{30}$ Trisubstituted olefin 6 reacted cleanly in the presence of cyclopropene A4 (entry 11). Surprisingly, in the absence of activator very high RCM selectivity was observed (entry 9). This illustrates the unpredictable behavior of Ru-6 in the absence of activator, which often results in mixtures of metathesis, cycloisomerization and isomerization products (e.g. entries 13, 17 and 21) but in some cases, can also afford relatively clean reactions (entries 9 and 33). Interestingly, 6-membered RCM product 9 was obtained in high yields independent on the source of activator (entries 14-16). RCM reactions with substrate $\mathbf{1 0}$ were carried out either under standard conditions with non-degassed toluene (entries 17-20) or using degassed toluene (entries 21-24). In general yields and conversions were similar in the two cases. However, we found the reaction with activator $\mathbf{A} 7$ to be more selective towards the metathesis product 11 with degassed toluene (entries 20 and 24). For the synthesis of macrocycle $\mathbf{1 3}$ we noticed that the use of degassed toluene gave better reproducibility compared to standard conditions. The yields were somewhat lower compared to 7-membered cycle 11 (entries 25-28), very likely due to the formation of oligomeric side products. ${ }^{31}$ Surprisingly, activators $\mathrm{A} 1$ and $\mathrm{A} 7$ performed significantly better than A4 (entries 26-28). Cross-metathesis reactions between 14 and $\mathbf{1 5}$ were very sluggish and low yielding (entries 29-32). We noticed that upon addition of Ru-6 to a mixture of $\mathbf{1 4}$ and $\mathbf{1 5}$ a black slimy residue appeared within seconds. The observed degradation might be similar to the corresponding $\mathrm{PCy}_{3}$ ligated complex which was reported to decompose in the presence of allylic alcohols. ${ }^{32}$

Table 1. Reaction scope. ${ }^{a}$ 


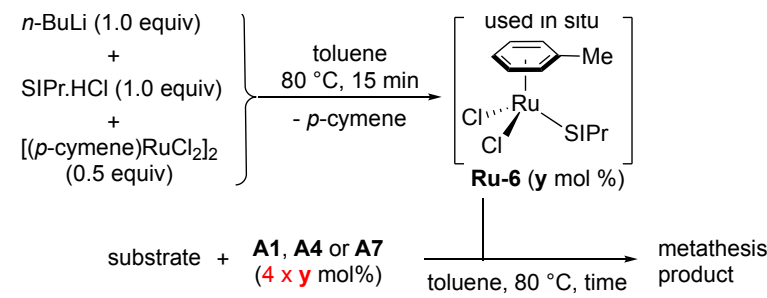

\begin{tabular}{|c|c|c|c|c|c|c|}
\hline Entry & Substrate & Product & $\begin{array}{c}\text { Ru-6 } \\
\text { (y mol \%) }\end{array}$ & $A X$ & $\begin{array}{l}\text { Time } \\
(\mathrm{min})\end{array}$ & $\begin{array}{c}\text { conv. (\%) / } \\
\text { yield (\%) }\end{array}$ \\
\hline 1 & & & 2 & none & 15 & $45 / 36^{b}$ \\
\hline 2 & & & 2 & A1 & 15 & $>98 / 91$ \\
\hline 3 & 10 & & 2 & A4 & 15 & $97 / 89$ \\
\hline 4 & & & 2 & A7 & 15 & $55 / 64^{b}$ \\
\hline $5^{c}$ & & & 2 & none & 15 & $18 / 12$ \\
\hline $6^{c}$ & 4 & 5 & 2 & A1 & 15 & $23 / 18$ \\
\hline $7^{c}$ & & & 2 & A4 & 15 & $90 / 81$ \\
\hline $8^{c}$ & & & 2 & A7 & 15 & $29 / 22$ \\
\hline 9 & & & 3 & none & 120 & $19 / 18$ \\
\hline 10 & & & 3 & A1 & 120 & $76 / 73$ \\
\hline 11 & & & 3 & A4 & 120 & $>98 / 95$ \\
\hline 12 & 6 & 7 & 3 & A7 & 120 & $92 / 86$ \\
\hline 13 & & & 3 & none & 120 & $>98 / 24^{b}$ \\
\hline 14 & & & 3 & A1 & 120 & $>98 / 98$ \\
\hline 15 & $=C C$ & & 3 & A4 & 120 & $>98 / 99$ \\
\hline 16 & 8 & 9 & 3 & A7 & 120 & $96 / 88^{b}$ \\
\hline 17 & & & 3 & none & 240 & $84 / 27^{b}$ \\
\hline 18 & & & 3 & A1 & 240 & $88 / 78$ \\
\hline 19 & & & 3 & A4 & 240 & $88 / 74$ \\
\hline 20 & & & 3 & A7 & 240 & $84 / 62^{b}$ \\
\hline $21^{d}$ & $\mathrm{E}=\mathrm{CO}_{2} \mathrm{Et}$ & & 3 & none & 240 & $91 / 6^{b}$ \\
\hline $22^{d}$ & 10 & 11 & 3 & A1 & 240 & $88 / 81$ \\
\hline $23^{d}$ & 10 & 11 & 3 & A4 & 240 & $90 / 72$ \\
\hline $24^{d}$ & & & 3 & A7 & 240 & $93 / 81$ \\
\hline
\end{tabular}

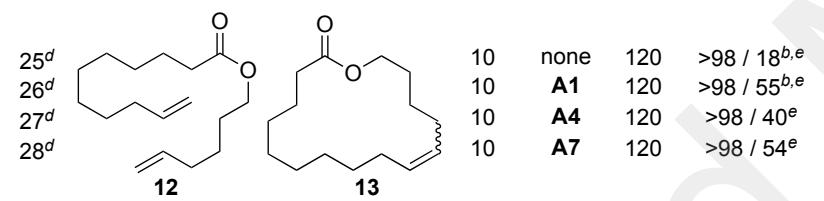

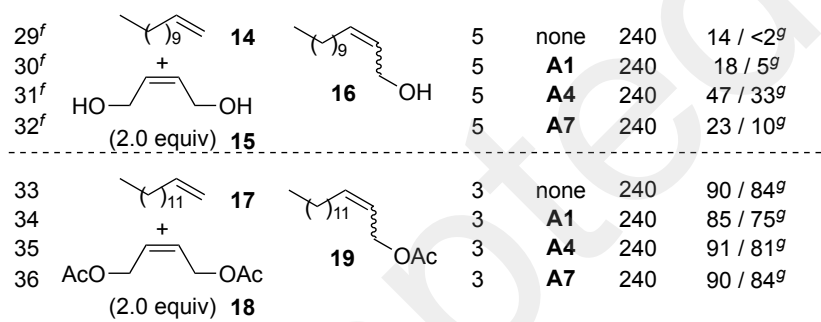

${ }^{a}$ Reactions carried out in argon-flushed vials with dry solvents (MBraun ${ }^{\circledR}$ solvent system). Conversions and yields were determined by GC-analysis with internal standards (see SI). ${ }^{b}$ Significant amounts of side products from cycloisomerization and isomerization processes were observed (see SI). ${ }^{c}$ Reactions carried out at room temperature. ${ }^{d}$ Reactions carried out in the glove-box with degassed solvents. ${ }^{e}$ Low yield due to oligomeric side products. $f \mathbf{R u}-\mathbf{6}$ was synthesized in toluene and then added to a solution of 14 and 15 in THF. Reactions in toluene afforded even lower yields. ${ }^{g}$ Apart from the desired products (16 or 19) we observed products from isomerization and self-metathesis of the terminal alkene substrates (14 or 17).

This assumption gains further credence when compared to the reactions with diacetate 18 which afforded CM product 19 in good yields with all activators (entries 33-36). Having tested several RCM and cross-metathesis reactions with substrates containing terminal olefins, we then decided to focus our attention on the self-metathesis of methyl oleate (i.e. methyl (Z)-9-octadecenoate) (Z)-20 (99\% purity) which is an important chemical feedstock from biomass, leading to valuable hydrocarbon $\mathbf{2 1}$ and mono-unsaturated diester $\mathbf{2 2}$ (Table 2). ${ }^{33}$ Surprisingly, activators like phenyl acetylene A1 and cyclopropene A4, which were among the best activators for RCM or CM reactions with terminal alkenes, gave poor or no conversion in the self-metathesis of (Z)-20 (entries 3 and 6). In contrast, when the reaction was carried out in the absence of activator the self-metathesis still proceeded, however producing many isomerization side-products (entry 1). The best productivity was found at low catalyst loading in the presence of diazocompounds A7 and A8 (entries 9-14). The reasons for the dramatic decrease of activity with alkyne and cyclopropene activators are still unclear. We postulate that the intermediate $\mathbf{E}$ generated from phenyl acetylene (Scheme 1a) is too sterically encumbered to efficiently react with the internal olefin of $(Z)$-20. In contrast, steric arguments cannot be evoked to explain the lack of efficiency with cyclopropene A4. In this case, the sterically more demanding cyclopropene A5 shows in fact better efficiency (entries 6-7). However, an electronic argument might be considered: Internal alkenes are more electron rich compared to terminal alkenes. Therefore, we postulate that electron poor cyclopropenes $\mathbf{A 5}$ and $\mathbf{A 6}$ lead to a better electronic match for in situ generated ruthenium catalyst with (Z)-20. On the other hand, we observed that, at high catalyst loading, the in situ generated ruthenium catalyst produces significant amounts of isomerization side-product (entries 9 and 13; see SI). Fortunately, lowering the catalyst loading to less than $500 \mathrm{ppm}$ favors the metathesis reaction over undesired isomerization (entries 10-12 and 14).

Table 2: Screening of activators for the self-metathesis of methyl (Z)-9-octadecenoate (Z)-20 (99\% purity) ${ }^{\mathrm{a}}$

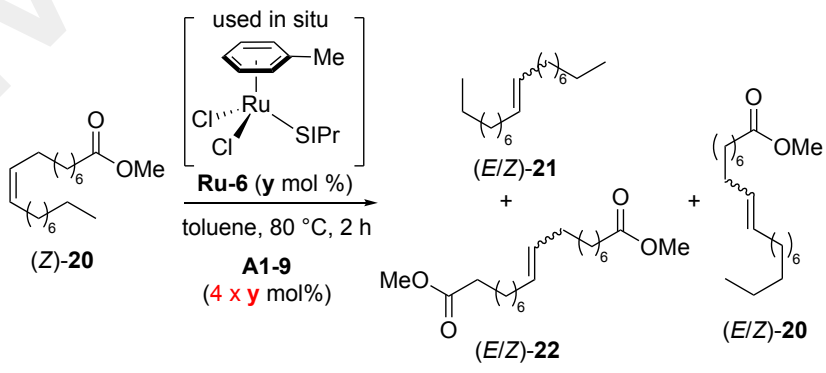

\begin{tabular}{|lccrcr|}
\hline Entry & $\begin{array}{c}\text { Ru-6 } \\
(\mathrm{y} \text { mol \%) }\end{array}$ & AX & $\begin{array}{c}\mathbf{2 0}(\%)^{b} \\
(Z: E \text { ratio })\end{array}$ & $\mathbf{2 1}(\%)^{b}$ & $\begin{array}{r}\mathbf{2 2}(\%)^{b} \\
(Z: E \text { ratio })\end{array}$ \\
\hline 1 & 2 & none & $36(21: 79)$ & 15 & $15(25: 75)$ \\
$2^{c}$ & 0.1 & none & $93(59: 41)$ & 3 & $3(32: 68)$ \\
3 & 2 & A1 & $100(>99: 1)$ & 0 & 0 \\
4 & 2 & A2 & $100(>99: 1)$ & 0 & 0 \\
5 & 2 & A3 & $100(>99: 1)$ & 0 & 0 \\
6 & 2 & A4 & $92(82: 18)$ & 4 & $4(30: 70)$ \\
7 & 2 & A5 & $51(24: 76)$ & 20 & $21(22: 78)$ \\
8 & 2 & A6 & $68(35: 65)$ & 16 & $16(24: 76)$ \\
9 & 2 & A7 & $38(21: 79)$ & 18 & $18(25: 75)$ \\
$10^{c}$ & 0.05 & A7 & $51(21: 79)$ & 24 & $24(23: 77)$ \\
$11^{d}$ & 0.02 & A7 & $51(21: 79)$ & 24 & $25(22: 78)$ \\
$12^{d}$ & 0.01 & A7 & $55(30: 70)$ & 22 & $23(24: 76)$ \\
13 & 2 & A8 & $15(13: 87)$ & 19 & $16(19: 81)$ \\
$14^{c}$ & 0.05 & A8 & $50(21: 79)$ & 24 & $24(24: 76)$ \\
15 & 2 & A9 & $72(32: 68)$ & 14 & $14(27: 73)$
\end{tabular}

${ }^{a}$ Reactions performed in argon flushed vials on a $0.3 \mathrm{mmol}$ scale (MO of $99 \%$ purity, Sigma Aldrich; used as received). The other products observed are unidentified isomerization products; see SI. ${ }^{b}$ Determined by GC. ${ }^{c}$ Carried out on a $8.0 \mathrm{mmol}$ scale. ${ }^{d}$ Reactions were carried out in the glove box (See SI for details).

When working with degassed solvents in a glove box, we even achieved $45 \%$ productive conversion $(50 \%$ being the 
maximum) with only $100 \mathrm{ppm}$ of in situ generated Ru-6 and $400 \mathrm{ppm}$ of activator $\mathbf{A 7}$ (entry 12). It is noteworthy that this is currently the best effective turn over number $(\mathrm{TON}=4500)$ achieved with in situ generated ruthenium catalysts. ${ }^{13}$ This TON is even higher compared to effective TON previously achieved with the Grubbs-1 catalyst Ru-1 $(\mathrm{TON}=2500) .{ }^{34}$ To demonstrate the robustness of in situ generated catalyst Ru-6, we tested the optimized catalytic system towards a non-refined methyl oleate Z-20 (technical grade, $85 \%$ purity) derived from very high oleic sunflower oils (VHOSO) on a 2-kg scale (Scheme 2). We were delighted that with only $400 \mathrm{ppm}$ of catalyst full equilibrium of $\mathbf{2 0}, \mathbf{2 1}$ and $\mathbf{2 2}$ was achieved within only 1 hour at $80{ }^{\circ} \mathrm{C}$ (See SI for a detailed description of the experiments including pictures). Of note, due to the low cost of $\left[(p \text {-cymene }) \mathrm{RuCl}_{2}\right]_{2}, \quad n$-BuLi, SIPr.HCl and ethyl diazoacetate A7, this catalytic system showing a TON of 1250 appeared quite competitive to the most efficient well-defined Ru-catalysts. ${ }^{33}$

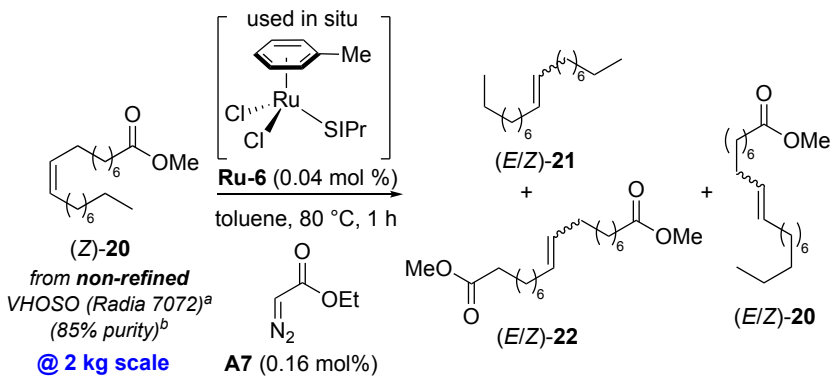

\begin{tabular}{l|ll} 
olefin distribution @ equilibrium & $c:$ & $\begin{array}{ll}\mathbf{2 0} & 42 \%(Z: E 21: 79) \\
\mathbf{2 1} & 21 \%(Z: E 21: 79)\end{array} \mid$
\end{tabular}

$2221 \%(Z: E$ 21:79)

Scheme 2. Kg-scale self-metathesis of non-refined methyl oleate (Z)-20 (technical grade, $85 \%$ purity) catalyzed by optimized in situ catalytic system Ru-6/A7

${ }^{a}$ Radia 7072 is commercialized by Oleon. ${ }^{b}$ The other compounds were identified as saturated and mono- or poly-unsaturated fatty methyl esters: C16:0 (3.4 wt.\%); C16:1 (0.1 wt.\%); C18:0 (1.8 wt.\%); C18:2 (3.1 wt.\%); C18:3 (1.8 wt.\%); C20:0 (0.2 wt.\%) C22:0 (0.3 wt.\%) and C22:1 (0.7 wt.\%). ${ }^{c}$ Determined by GC.

In conclusion, we have reported a systematic study concerning the in situ formation of Ru-alkylidene catalytic species for olefin metathesis. By combining commercially available and inexpensive reagents $\left[\mathrm{Ru}(p \text {-cymene }) \mathrm{Cl}_{2}\right]_{2}$, SIPr.HCl and $n$-BuLi in toluene, the resulting 18 electron arene-ruthenium complex promoted, in the presence of activators such as alkynes, cyclopropenes and diazoesters, highly selective and efficient catalytic systems toward a variety of olefin metathesis transformations. Furthermore, we introduced for the first time cyclopropenes as efficient activators for the in situ generation of Ru-metathesis catalysts. Our investigations concerning the self-metathesis of methyl oleate (MO) showed an unprecedented substrate dependence on the activators. The diazoester $\mathbf{A} 7$ was identified to be most efficient to convert the MO (Z)-20 (@99\% purity) into highly valuable mono-unsaturated diester and hydrocarbon with a catalyst loading as low as $100 \mathrm{ppm}$ (TON of 4500). Moreover, the robustness of the newly developed catalytic system was demonstrated with the $2 \mathrm{~kg}$-scale transformation of a nonrefined technical grade MO (Z)-20 (85\% purity) using only $400 \mathrm{ppm}$ of catalyst loading $(\mathrm{TON}=1250)$. This highly competitive and cost-effective catalytic process is underway in other valuable metathesis transformations.

\section{ASSOCIATED CONTENT}

Supporting Information. Optimization reactions, experimental procedures and characterization data $\left({ }^{1} \mathrm{H} \mathrm{NMR},{ }^{13} \mathrm{C} \mathrm{NMR}, \mathrm{GC}-\right.$ traces). This material is available free of charge via the Internet at http://pubs.acs.org.

\section{AUTHOR INFORMATION}

\section{Corresponding Author}

*E-mail: marc.mauduit@ensc-rennes.fr

\section{ORCID}

Daniel S. Müller: 0000-0001-8184-1474

Olivier Baslé : 0000-0002-4551-473X

Marc Mauduit : 0000-0002-7080-9708

\section{Present Addresses}

$\dagger$ Laboratoire de Chimie de Coordination, CNRS, UPR 8241, 205 route de Narbonne, 31077 Toulouse Cedex 04, France

Notes

The authors declare no competing financial interests

\section{ACKNOWLEDGMENT}

This work was supported by the FASO (grant to DSM; Z-SELECT) and the Région Bretagne (SAD $2016 \mathrm{~N}^{\circ} 9639$ RZSELECT; grant to OB and DSM). OB and MM acknowledge the Ecole Nationale Supérieure de Chimie de Rennes (ENSCR) and the Centre National de la Recherche Scientifique (CNRS) for financial support. Umicore $\mathrm{AG} \& \mathrm{Co}$ is acknowledged for a generous gift of $\left[(p\right.$-cymene $\left.) \mathrm{RuCl}_{2}\right]$ complex.

\section{REFERENCES}

(1) Selected comprehensive books on olefin metathesis: (a) Handbook of Metathesis, 2nd ed.; Grubbs, R. H.; Wenzel, A. G.; O'Leary, D. J.; Khosravi E., Eds.; Wiley-VCH, Weinheim, Germany, 2015. (b) Olefin Metathesis: Theory and Pratice, Grela, K.; Wiley-VCH, Weinheim, Germany 2014.

(2) Schwab, P.; Grubbs, R. H.; Ziller, J. W. The Influence of the Alkylidene Moiety on Metathesis Activity. Synthesis and Applications of $\mathrm{RuCl}_{2}\left(=\mathrm{CHR}^{\prime}\left(\mathrm{PR}_{3}\right)_{2}\right.$. J. Am. Chem. Soc. 1996, 118, 100-110.

(3) For applications in industry, see: (a) Higman, C. S.; Lummiss, J. A. M.; Fogg. D. E. Olefin Metathesis at the Dawn of Implementation in Pharmaceutical and Specialty-Chemicals Manufacturing. Angew. Chem. Int. Ed. 2016, 55, 3552-3565. (b) Hughes, D.; Wheeler, P.; Ene, D. Olefin Metathesis in Drug Discovery and DevelopmentExamples from Recent Patent Literature. Org. Process Res. Dev. 2017, 21, 1938-1962. (c) Yu, M.; Lou, S.,; Gonzalez-Bobes, F. RingClosing Metathesis in Pharmaceutical Development: Fundamentals, Applications, and Future Directions. Org. Proc. Res. Dev. 2018, 22, 918-946. For applications in total synthesis, see: (d) Nicolaou, K. C.; Bulger, P. G.; Sarlah, D. Metathesis Reactions in Total Synthesis. Angew. Chem. Int. Ed. 2005, 44, 4490-4527. (e) Cheng-Sánchez, I.; Sarabia. F. Recent Advances in Total Synthesis via Metathesis Reactions. Svnthesis 2018, 50, 3749-3786.

(4) (a) Dias, E. L.; Nguyen, S. T.; Grubbs. R. H. Well-Defined Ruthenium Olefin Metathesis Catalysts: Mechanism and Activity. J. Am. Chem. Soc. 1997, 119, 3887-3897. (b) Scholl, M.; Ding, S.; Lee, C. W.; Grubbs, R. H. Synthesis and Activity of a New Generation of Ruthenium-Based Olefin Metathesis Catalysts Coordinated with 1,3-Dimesityl-4,5-dihydroimidazol-2-ylidene Ligand. Org. Lett. 1999, 1, 953-956. 
(5) For recent investigations concerning catalyst degradation, see: (a) Kadyrov, R. Low Catalyst Loading in Ring-Closing Metathesis Reactions. Chem. Eur. J. 2013, 19, 1002-1012. (b) Kadyrov, R. Olefin Metathesis: Catalyst Inhibition as a Result of Isomerization. ChemCatChem 2016, 8, 113-116. (c) McClennan, W. L.; Rufh, S. A; Lummiss, J. A. M.; Fogg. D. E. A General Decomposition Pathway for Phosphine-Stabilized Metathesis Catalysts: Lewis Donors Accelerate Methylidene Abstraction. J. Am. Chem. Soc. 2016, 138, 14668-14677. (d) Higman, C. S.; Lanterna, A. E.; Marin, M. L.; Scaiano, J. C.; Fogg, D. E. Catalyst Decomposition During Olefin Metathesis Yields Isomerization-Active Ruthenium Nanoparticles. ChemCatChem 2016, 8, 2446-2449. (e) Bailey, G. A.; Foscato, M.; Higman, C. S.; Day, C. S.; Jensen, V. R.; Fogg D. E. Bimolecular Coupling as a Vector for Decomposition of Fast-Initiating Olefin Metathesis Catalysts. J. Am. Chem. Soc. 2018, 140, 6931-6944. For a review discussing catalyst degradation, see: (f) Nelson, D. J.; Manzini, S.; Urbina-Blanco, C. A.; Nolan, S. P. Key Processes in Ruthenium-Catalysed Olefin Metathesis. Chem. Commun. 2014, 50, 10355-10375.

(6) (a) Demonceau, A.; Noels, A. F.; Saive, E. Hubert, A. J. Ruthenium-Catalysed Ring-Opening Metathesis Polymerization of Cycloolefins Initiated by Diazoesters. J. Mol. Cat. 1992, 76, 123-132. Closely related, $\mathrm{PPh}_{3}$ ligated catalysts were synthesized previously by Baird and Bennet: (b) Zelonka, R. A.; Baird. M. C. Benzene Complexes of Ruthenium(II). Canadian Journal of Chemistry, 1972, 50, 3063-3072. (c) Bennet, M. A.; Smith, A. K.; Arene Ruthenium(II) Complexes Formed by Dehydrogenation of Cyclohexadienes with Ruthenium(III) Trichloride. J. Chem. Soc. Dalton Trans., 1974, 0 , 233-241. Ru-2 is commercially available; CAS: 145381-23-3.

(7) Herrmann, W. A.; Elison, M.; Fischer, J.; Kocher, C.; Artus. G. R. J. $\mathrm{N}$-Heterocyclic Carbenes: Generation under Mild Conditions and Formation of Group 8-10 Transition Metal Complexes Relevant to Catalysis. Chem. Eur. J. 1996, 2, 772-780. Nolan was the first to report metathesis reactions with $\mathbf{R u - 3}$ (see ref. 8).

(8) Jafarpour, L.; Huang, J.; Stevens, E. D.; Nolan, S. P.; (p-cymene) $\mathrm{RuLCl}_{2} \quad(\mathrm{~L}=1,3$-Bis(2,4,6-trimethylphenyl)imidazol-2ylidene and 1,3-Bis(2,6-diisopropylphenyl)imidazol-2-ylidene) and Related Complexes as Ring Closing Metathesis Catalysts. Organometallics 1999, 18, 3760-3763.

(9) Engel, J.; Smit, W.; Foscato, M.; Occhipinti, G.; Törnroos, K. W.; Jensen V. R. Loss and Reformation of Ruthenium Alkylidene: Connecting Olefin Metathesis, Catalyst Deactivation, Regeneration, and Isomerization. J. Am. Chem. Soc. 2017, 139, 16609-16619.

(10) During the preparation of this manuscript Fogg reported an improved synthesis of Ru-5: Day, C. S.; Fogg D. E.; High-Yield Synthesis of a Long-Sought, Labile Ru-NHC Complex and Its Application to the Concise Synthesis of Second-Generation Olefin Metathesis Catalysts. Organometallics, 2018, 37, 4551-4555.

(11) For an excellent review concerning Ru-arene catalysts, see: Delaude, L.; Demonceau, A. Retracing the Evolution of Monometallic Ruthenium-Arene Catalysts for $\mathrm{C}-\mathrm{C}$ Bond Formation. Dalton Trans. 2012, 41, 9257-9268.

(12) a) Demonceau, A.; Stumpf, A. W.; Saive, E.; Noels, A. F.; Novel Ruthenium-Based Catalyst Systems for the Ring-Opening Metathesis Polymerization of Low-Strain Cyclic Olefins. Macromolecules 1997, 30, 3127-3136. (b) Jan, D.; Delaude, L.; Simal, F.; Demonceau, A. Noels, A. F. Synthesis and Evaluation of New $\mathrm{RuCl}_{2}(p-$ cymene $)\left(E_{2} R^{\prime}\right)$ and $\left(\eta^{1}: \eta^{6}\right.$-phosphinoarene $) \mathrm{RuCl}_{2}$ Complexes as Ring-Opening Metathesis Polymerization Catalysts J. Organomet. Chem. 2000, 606, 55-64. (c) Baran, J.; Bogdanska, I.; Jan, D.; Delaude, L.; Demonceau, A.; Noels, A. F.. Synthesis and RingOpening Metathesis Polymerization of Eight-Membered Unsaturated Lactams and Related Monomers. J. Mol. Cat. A: Chemical 2002, 190, 109-116. (d) Delaude, L.; Demonceau, A.; Noels A. F. Synthesis and Application of New N-Heterocyclic Carbene Ruthenium Complexes in Catalysis: A Case Study. Curr. Org. Chem. 2006, 10, 203-215.

(13) Ahr, M.; Thieuleux, C.; Copéret, C.; Fenet, B.; Basseta J.-M. Noels' vs. Grubbs' Catalysts: Evidence for One Unique Active Species for Two Different Systems. Adv. Svnth. Catal. 2007, 349, 1587-1591.
(14) (a) Louie, J.; Grubbs, R. H. Highly Active Metathesis Catalysts Generated In Situ from Inexpensive and Air-Stable Precursors. Angew. Chem. Int. Ed. 2001, 40, 247-249. b) Sémeril, D.; Bruneau, C.; Dixneuf, P. H. Ruthenium Catalyst Dichotomy: Selective Catalytic Diene Cycloisomerization or Metathesis. Helv. Chim. Acta 2001, 84, 3335-3341. c) Sauvage, X.; Borguet, Y.; Noels, A. F.; Delaude, L.; Demonceau, A. Homobimetallic Ruthenium- $N$ Heterocyclic Carbene Complexes: Synthesis, Characterization, and Catalytic Applications. Adv. Synth. Catal. 2007, 349, 255-265. d) Lo, C.; Cariou, R.; Fischmeister, C.; Dixneuf, P. H. Simple Ruthenium Precatalyst for the Synthesis of Stilbene Derivatives and Ring-Closing Metathesis in the Presence of Styrene Initiators. Adv. Synth. Catal. 2007, 349, 546-550.

(15) (a) Fürstner, A.; Ackermann, L. A. Most User-Friendly Protocol for Ring-Closing Metathesis Reactions. Chem. Commun. 1999, 9596. (b) Fürstner, A.; Müller, T. Efficient Total Syntheses of Resin Glycosides and Analogues by Ring-Closing Olefin Metathesis. J. Am. Chem. Soc. 1999, 121, 7814-7821. (c) Fürstner, A.; Thiel, O. R. Formal Total Synthesis of (-)-Balanol: Concise Approach to the Hexahydroazepine Segment Based on RCM. J. Org. Chem. 2000, 65, $1738-1742$

(16) (a) Delaude, L.; Demonceau, A.; Noels A. F. Visible Light Induced Ring-Opening Metathesis Polymerisation of Cyclooctene. Chem. Commun. 2001, 986-987. (b) Delaude, L.; Szypa, M.; Demonceau, A.; Noels, A. F. New In Situ Generated Ruthenium Catalysts Bearing N-Heterocyclic Carbene Ligands for the Ring-Opening Metathesis Polymerization of Cyclooctene. Adv. Synth. Catal. 2002, 344, 749-756.

(17) (a) Lübbe, C.; Dumrath, A.; Neumann, H.; Beller, M.; Kadyrov R. Lewis Acid Assisted Ruthenium-Catalyzed Metathesis Reactions. ChemCatChem 2014, 6, 105-108. Concerning the important role of impurities, see: b) Lübe, C.; Dumrath, A.; Neumann, H.; Schäffer, M.; Zimmermann, R.; Beller, M.; Kadyrov, R. How Important are Impurities in Catalysis? An Example from Ring-Closing Metathesis. ChemCatChem 2014, 6, 684-688.

(18) Noels reported twice the use of isolated complex Ru-5 but no characterization of the complex was given: a) Stumpf, A. W.; Saive, E.; Demonceau, A.; Noels, A. F. Ruthenium-Based Catalysts for the Ring-Opening Metathesis Polymerisation of Low-Strain Cyclic Olefins and of Functionalised Derivatives of Norbornene and Cyclooctene. Chem. Commun. 1995, 1127-1128 and ref. 12a. For a failed attempt to synthesize Ru-5, see: c) Ledoux, N.; Allaert, B.; Verpoort F. Ruthenium-Based NHC-Arene Systems as Ring-Opening Metathesis Polymerisation Catalysts. Eur. J. Inorg. Chem. 2007, 5578-5583. The first succesful synthesis, albeit in low yield (26\%) of Ru-5 was reported by Occhipinti and Jensen, see ref. 9 .

(19) Grubbs already reported that $\left(\left[\mathrm{RuCl}_{2}\left(\mathrm{PPh}_{3}\right)_{3}\right]\right.$ and $\left[\left\{\mathrm{RuCl}_{2}(\mathrm{cod})\right\}_{\mathrm{n}}\right]$ were unsuitable precursors, see ref. 14a.

(20) See Supporting Information (SI) for additional screening experiments.

(21) ESI mass spectrometry the Ru-stock solution confirmed the presence of Ru-6 (see SI).

(22) A4 can be easily prepared on large scale, see: (a) Sherrill, W. M.; Kim, R.; Rubin, M. Improved Preparative Route Toward 3-Arylcyclopropenes. Tetrahedron 2008, 64, 8610-8617. Cyclopropenes show similar reactivity than alkynes: (b) Müller, D. S.; Marek, I.; Copper Mediated Carbometalation Reactions. Chem. Soc. Rev. 2016, 45, 4552-4566. (c) Müller, D. S.; Marek, I. Asymmetric Copper-Catalyzed Carbozincation of Cyclopropenes en Route to the Formation of Diastereo- and Enantiomerically Enriched Polysubstituted Cyclopropanes. J. Am. Chem. Soc. 2015, 137, 15414-15417. (d) Müller, D. S.; Werner, V.; Akyol, S.; Schmalz, H. G.; Marek, I. Tandem Hydroalumination/Cu-Catalyzed Asymmetric Vinyl Metalation as a New Access to Enantioenriched Vinylcyclopropane Derivative. Org. Lett. 2017, 19, 3970-3973.

(23) Grünwald, C.; Gevert, G.; Wolf, J.; Bonzález-Herrero, P.; Werner, H. Five-Coordinate 16-Electron Carbene- and Vinylideneruthenium(II) Complexes Prepared from $\left[\mathrm{RuCl}_{2}\left(\mathrm{C}_{8} \mathrm{H}_{12}\right)\right]_{\mathrm{n}}$ 
or from the New Dihydridoruthenium (IV) Compound $\left[\mathrm{RuH}_{2} \mathrm{Cl}_{2}\left(\mathrm{P}^{i} \mathrm{Pr}_{3}\right)_{2}\right]$. Organometallics 1996, 15, 1960-1962.

(24) (a) Katayama, H.; Ozawa F. Convenient Routes to Vinylideneruthenium Dichlorides with Basic and Bulky Tertiary

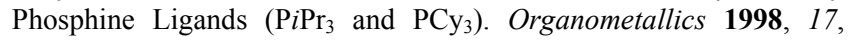
5190-5196. (b) For a mechanistic study concerning the formation of metal vinylidenes from alkynes, see: Wakatsuki, Y.; Mechanistic Aspects Regarding the Formation of Metal Vinylidenes from Alkynes and Related Reactions. J. Organomet. Chem. 2004, 689, 4092-4109.

(25) (a) Borguet, Y.; Sauvage, X.; Zaragoza, G.; Demonceau, A.; Delaude, L. Homobimetallic Ruthenium-Arene Complexes Bearing Vinylidene Ligands: Synthesis, Characterization, and Catalytic Application in Olefin Metathesis. Organometallics 2010, 29, 66756686. (b) Borguet, Y.; Sauvage, X.; Zaragoza, G.; Demonceau, A.; Delaude, L. Synthesis and Catalytic Evaluation in Olefin Metathesis of a Second-Generation Homobimetallic Ruthenium-Arene Complex Bearing a Vinylidene Ligand. Organometallics 2011, 30, 2730-2738.

(26) Johnson, L. K.; Grubbs, R. H.; Ziller, J. W. Synthesis of Tungsten Vinyl Alkylidene Complex via the Reactions of $\mathrm{WCl}_{2}(\mathrm{NAr})\left(\mathrm{PX}_{3}\right)_{3}(\mathrm{X}=\mathrm{R}, \mathrm{OMe})$ Precursors with 3,3-Disubstituted Cyclopropenes. J. Am. Chem. Soc. 1993, 115, 8130-8145.

(27) Nguyen, S. T.; Johnson, L. K.; Grubbs R. H. Ring-Opening Metathesis Polymerization (ROMP) of Norbornene by a Group VIII Carbene Complex in Protic Media. J. Am. Chem. Soc. 1992, 114, 3974-3975.

(28) Noels demonstrated previously that it is important to add the ruthenium-arene complex to the activator and the substrate, see ref. 6 a.

(29) Galardon, E.; Le Maux, P.; Toupet, L.; Simonneaux, G. Synthesis, Crystal Structure, and Reactivity of $(5,10,15,20$
Tetraphenylporphyrinato)ruthenium(II) (Diethoxycarbonyl)carbene Methanol. Organometallics 1998, 17, 565-569.

(30) Grela reported that sterically encumbered indenylidene catalysts showed much higher catalytic activity at $70{ }^{\circ} \mathrm{C}$ compared to $30{ }^{\circ} \mathrm{C}$ : Bieniek, M.; Michrowska, A.; Usanov, D. L.; Grela, K. In an Attempt to Provide a User's Guide to the Galaxy of Benzylidene, Alkoxybenzylidene and Indenylidene Ruthenium Olefin Metathesis Catalysts. Chem. Eur. J. 2008, 14, 806-818.

(31) Oligomerization products are commonly observed in RCM reactions producing macrocycles, for example: Müller, D. S.; Curbet, I.; Raoul, Y.; Le Nôtre, J.; Baslé, O.; Mauduit, M. Stereoretentive Olefin Metathesis Made Easy: In Situ Generation of Highly Selective Ruthenium Catalysts from Commercial Starting Materials. Org. Lett. 2018, 20, 6822-6826.

(32) Solari, E.; Gauthier, S.; Scopelliti, R.; Severin K. Multifaceted Chemistry of $\left[(p \text {-cymene }) \mathrm{RuCl}_{2}\right]_{2}$ and $\mathrm{PCy}_{3}$. Organometallics 2009, $28,4519-4526$.

(33) Allard, J.; Curbet, I.; Chollet, G.; Tripoteau, F.; Sambou, S.; Caijo, F.; Raoul, Y.; Crévisy, C.; Baslé, O.; Mauduit, M. Bleaching Earths as Powerful Additives for Ru-Catalyzed Self-Metathesis of Non-Refined Methyl Oleate at Pilot Scale. Chem. Eur. J. 2017, 23, 12729-12734.

(34) Dinger, M. B.; Mol, J. C. High Turnover Numbers with Ruthenium-Based Metathesis Catalysts. Adv. Svnth. Catal. 2002, 344, 671-677. 


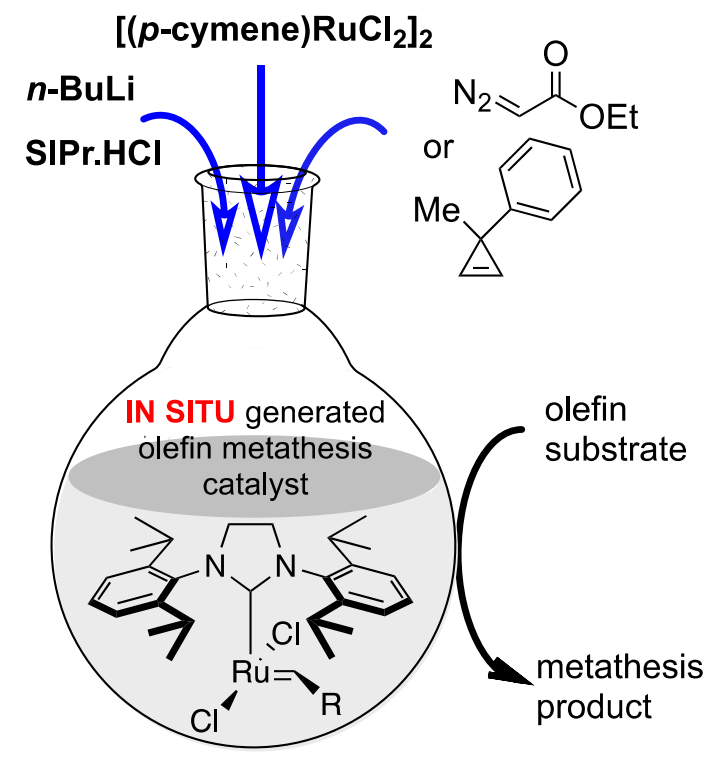

Commercially available \& inexpensive starting materials

Bench procedure (argon-flushed)

Catalyst loading as low as $100 \mathrm{ppm}$ (TON of 4500)

\section{9 examples (RCM \& CM)}

Transformation of non-refined methyl oleate ( $85 \%$ purity) @ 2 kg-scale Robust \& cost-effective catalytic system 